\title{
Life threatening plexiform neurofibroma of a young child
}

\author{
Edit Bardi ${ }^{1}$, Peter Masat ${ }^{1}$, Mihaly Kisely ${ }^{2}$, J anos Huszka $^{3}$, Balazs Tolvaj ${ }^{4}$, Ferenc Garzuly ${ }^{4}$, \\ Bernadette Kalman ${ }^{5,6}$
}

1. Department of Pediatrics, Markusovszky University Teaching Hospital, Szombathely, Hungary. 2. Department of Ear, Nose and Throat, Markusovszky University Teaching Hospital, Szombathely, Hungary. 3. Department of Ear, Nose, Throat, and Head and Neck Surgery, Peterfy Sandor Hospital and Outpatient Clinic, Budapest, Hungary. 4. Department of Pathology, Markusovszky University Teaching Hospital, Szombathely, Hungary. 5. Center for Research and Education, Markusovszky University Teaching Hospital, Szombathely, Hungary. 6. University of Pecs, Pecs, Hungary.

Correspondence: Bernadette Kalman. Address: University of Pecs, Markusovszky University Hospital, 5. Markusovszky Street, 9700. Szombathely, Hungary. E-mail: kalman.bernadett@markusovszky.hu

Received: July 14, 2014

Accepted: September 9, 2014

Online Published: September 17, 2014

DOI : $10.5430 / c r c p . v 2 n 1 p 34$

URL: http://dx.doi.org/10.5430/crcp.v2n1p34

\section{Abstract}

Aim: To report a child with an extensive tumor invading multiple anatomical structures within the left side of her neck.

Material and methods: Presentation of a case including patient's history, physical exam, molecular data, MRI imaging, histopathology and molecular genetics.

Results: The life-threatening compression as well as the dislocation of the trachea required meticulous surgical removal of the tumor. Histology revealed a plexiform neurofibroma related to a frame shift mutation in the NF1 gene.

Conclusion: Surgical removal of this extensive tumor saved the patient's life. The long term success of management will be related to the effectiveness of the maintenance therapy that currently includes interferon- $\alpha 2 \mathrm{a}$. This report highlights unique aspects of a rare but life threatening condition in a young girl.

\section{Key words}

Plexiform neurofibroma, Neurofibromatosis-1, Surgery, Therapy

\section{I ntroduction}

Neurofibromatosis-1 (NF-1) and neurofibromatosis-2 (NF-2) are distinguished based on clinical, imaging, pathological and molecular genetic characteristics. Plexiform neurofibroma is a presentation of NF-1, which typically grows along multiple nerve fascicles and occurs in childhood. Its size may greatly vary. Larger tumors can be highly invasive and cause deformities of affected body parts or life-threatening conditions by compressing vital anatomical structures. Surgical removal is the first line of intervention that, however, can only be partial or subtotal in most cases ${ }^{[1]}$. Medical maintenance therapy and long term tumor monitoring are essential ${ }^{[2-4]}$. We present a small child with a large cervical plexiform neurofibroma that caused both diagnostic and therapeutic difficulties, and discuss that even after a successful surgical resection of the tumor, long term management of such a case may still involve challenges. 


\section{Materials and methods}

We review the medical history, physical exam and MRI imaging of a four years old girl with a tumor invading the blood vessels, nerves and muscles in the left side of her neck, and compressing as well as dislocating the trachea. After surgical removal, histological and immunohistochemistry (IHC) analyses of the tumor were performed. Exons of the NF1 gene were analyzed by direct sequencing of the patient's blood-derived DNA.

\section{Results}

\subsection{Medical history}

The girl was born at 38 weeks of pregnancy and had a normal postnatal period. From six months of age, her somatic development showed signs of delay. At three years of age, in May 2013, she was referred to our hospital with a long standing "cervical lymphadenitis". Her family history was negative. The parents noted a left cervical growth half a year before.

\subsection{Clinical exams and diagnostic work up}

The patient's physical exam was normal with the exception of the cervical tumor, pectus excavatum, several "café au lait" spots on her trunk and axillary freckles. Cardiologic and ophthalmologic exams were non-revealing. Routine blood counts, liver and kidney functions, serum electrolytes, ferritin, VMA, CEA, AFP and HCG and her bone marrow aspirate were negative. Serology was also negative for Borrelia burgdorferi, Bartonella, cytomegalovirus, Epstein-Barr virus, hepatitis A, B and C, Human Immunodeficiency Virus, Toxoplasma and Toxocara. EEG was normal. Based on an ultrasound exam, the cervical growth was interpreted as enlarged lymph nodes. Doppler of the kidneys and the major blood vessels showed normal vascular structures.

MRI of the neck (see Figure 1a, 1b) showed an extensive, multi-lobular tumor invading all blood vessels, nerves and muscles on the left side of the neck, and compressing as well as dislocating the trachea and the larynx. The MRI of the brain showed no space-occupying lesion, but T2-weighted and FLAIR sequences depicted multiple small hyperintense spots (not shown).

Histology of a tumor biopsy taken in August 2013 established the diagnosis of plexiform neurofibroma (not shown).

Sequencing 58 exons of the NF1 gene revealed two known single nucleotide polymorphisms and a novel, heterozygous single nucleotide deletion at position c.5190 in exon 28.2. The latter represents a frame shift mutation and is the likely cause of pathology.

\subsection{Surgical intervention}

Since the tumor caused progressive respiratory difficulties, its removal seemed necessary, but due to the extensive infiltration of vital tissue regions, the consulting outside surgical team suggested initially medical therapy. However, as the tumor continued to grow and the patient's breathing became even more compromised, the local ear, nose and throat (ENT) specialist (MK) along with an invited ENT \& head and neck surgeon $(\mathrm{JH})$ performed the challenging, six-hour-long operation in intratracheal narcosis, in November 2013. After the dermal dissection extending from the tip of left mastoid bone to the cricoid cartilage, and underneath of the platysma, multiple tumorous nodes ranging in the size of half a millimeter to 3-4 centimeters were visible (see Figure 1c). First, the invading lesions were successfully removed from the wall of the internal jugular vein. The external carotid artery was too intimately invaded making its ligation necessary in the interest of tumor resection. Subsequently, parts of the tumor that protruded into the posterior wall of the pharynx and dislocated the larynx were removed. Resection of the invaded accessory nerve was inevitable, after which, removal of the extensive tumor continued from underneath the trapezius muscle and the supraclavicular region. The lower third part of 
the sternocleidomastoid muscle also had to be sacrificed due to the tumor invasion. After the completion of dissection, the wound was closed with a suction drainage in place.

Figure 1. MRI images and an intraoperative photograph of the plexiform neurofibroma

It shows coronal T1-weighted post-contrast (a) and T2-weighted (b) MRI images along with an intraoperative photograph (c) of the cervical tumor.

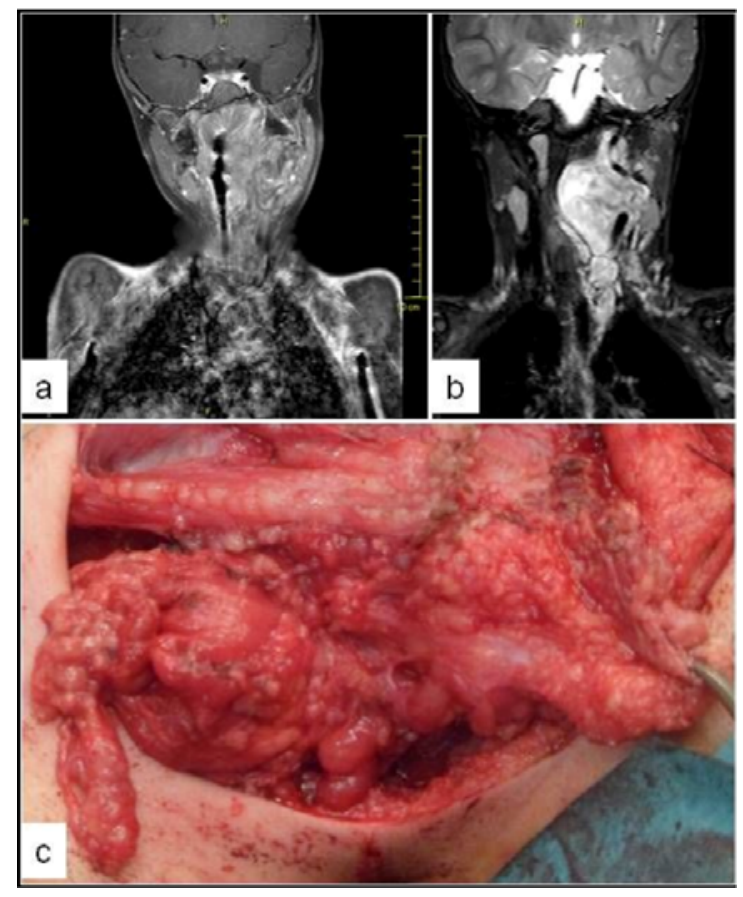

\subsection{Histological characteristics of the dissected tumor}

Figure 2a shows the resected plexiform tumor. Neither the surgical, nor the pathological dissection was able to determine, from which nerve's sheet the tumor originated. Figure $2 b$ and $c$ show hematoxylin-eosin staining and S-100 immunohistochemistry of a representative section depicting Schwann cells without malignant transformation and with low proliferation index (Ki 67 positivity was 2\%-3\%, not shown). Figure 3 demonstrates further histological components including the perineurial cells around enlarged fascicules (a), fragmented axons (b) irregular collagen fibers (c) and mucoid matrix (d) in the tumor.

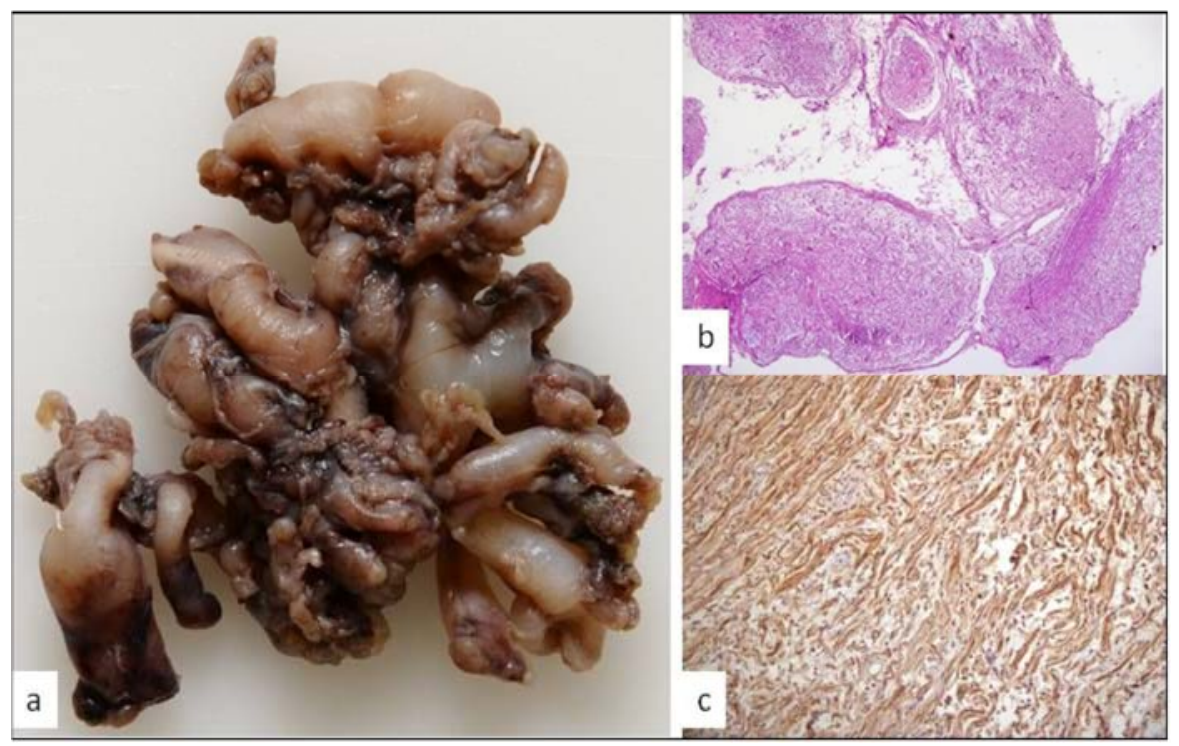

Figure 2. Gross pathology and histologic work up of the tumor. It shows gross pathology of the dissected plexiform tumor (a) along with hematoxylin-eosin staining (b) and S-100 immunohistochemistry (c) of tumor sections. The latter (c) reveals low rate of Schwann cell proliferation. 


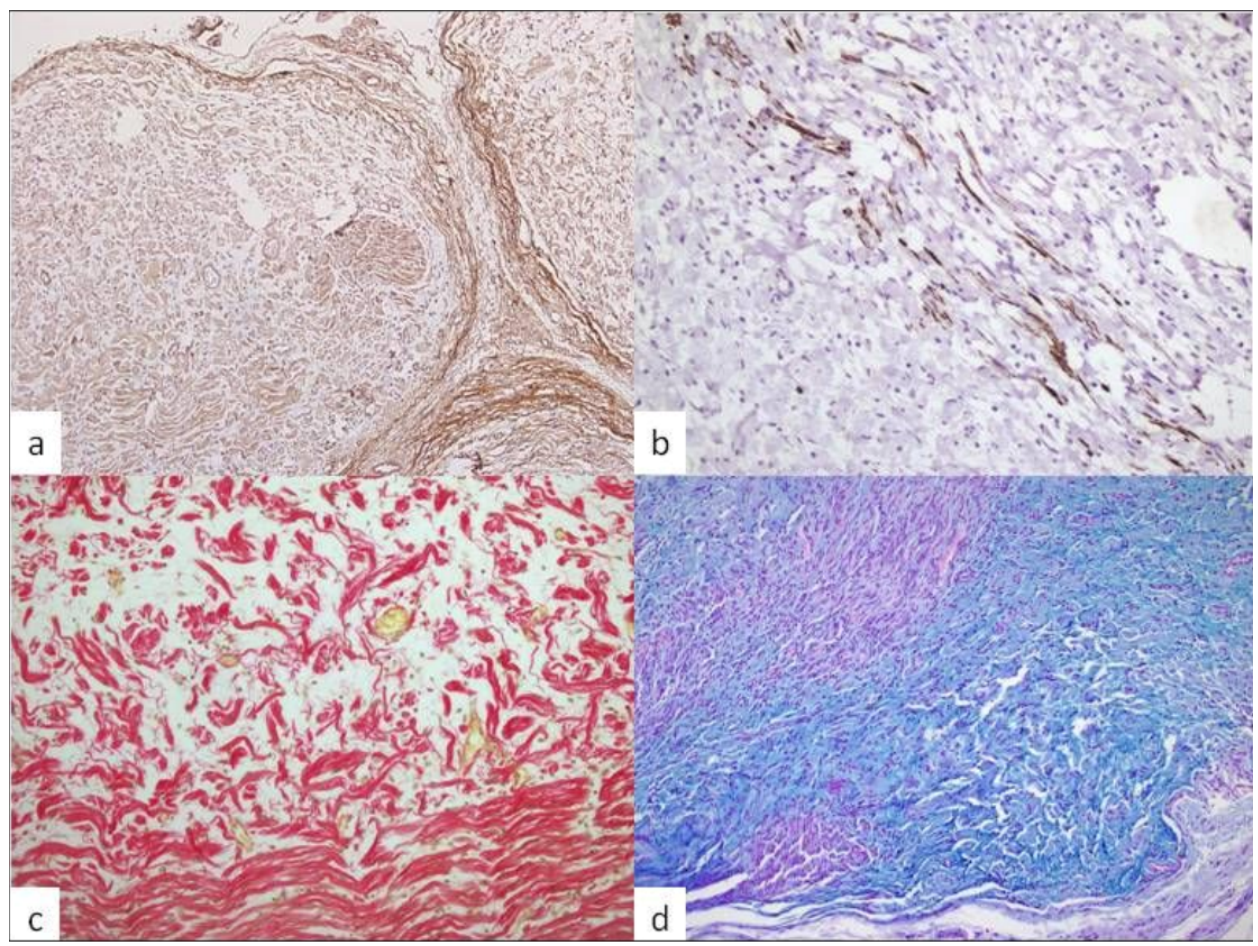

Figure 3. Histological contents of the tumor. It depicts perineurial cells around enlarged fascicules (epithelial membrane antigen - EMA IHC) (a), fragmented axons (neurofilament $68 \mathrm{kD} \mathrm{IHC)} \mathrm{(b),} \mathrm{"shredded} \mathrm{carrots"} \mathrm{collagen} \mathrm{fibers} \mathrm{(picrosirius}$ red staining) (c), and mucoid matrix within the tumor (alcian blue - PAS staining) (d).

\subsection{Current management}

The patient's breathing difficulties normalized after surgery. She is currently being treated with interferon $\alpha$, and monitored for tumor recurrence as well as for new tumor occurrence. Should interferon $\alpha$ be ineffective, imatinib will be considered as a rescue therapy.

\section{Discussion}

NF1 is a rare disease with a prevalence of 1:2700 ${ }^{[2]}$. The spectrum of its phenotypic presentation is wide, and may include a variety of benign and malignant tumors along with bone and other developmental abnormalities ${ }^{[2]}$. Neurofibromas are slowly growing, benign tumors that arise from nerve sheets and comprise Schwann cells, fibroblasts and perineurial cells. Plexiform neurofibroma is a distinct presentation within the spectrum of NF1 pathology, and may be seen either in combination with other tumors or alone as a solitary lesion. The latter is exemplified by our case (see Figures 1, 2 and 3). Cutaneous, intraneural and soft tissue variants have been described ${ }^{[5]}$. It, however, most often arises in body parts with rich innervations such as the face, orbit, neck, trunk or inguinal regions, and may reach a size that not only causes disfigurement but also jeopardizes the patient's life. Rarely, occurrence of plexiform neurofibroma has been observed in salivary glands ${ }^{[5]}$.

In our case, the cervical tumor imitated the appearance of lymphadenitis, and the correct diagnosis could be established only after MRI studies and histology work up of a tumor biopsy. The subsequent subtotal removal of the large cervical plexiform neurofibroma corrected the severely compromised respiration for our patient. However, the successful surgical intervention was only feasible by sacrificing some of the cervical muscles, nerves and the external carotid artery.

In the study by Nguyen et al. ${ }^{[4]}, 52$ patients with a wide age range $(3.2-64.2$ years) and plexiform neurofibroma were analyzed. Sixteen percent of patients had acute surgical complications, while 13 percent had late complications. Due to 
tumor progression, repeated surgery was necessary in eight patients. Younger patients experienced the highest progression rates, but tumor type, location and depth also defined progression after surgery. Complete tumor resection was feasible in 13 patients and resulted in no recurrence in the mean observation period of 2.9 years (range 1.1-5.8 years) ${ }^{[4]}$. Prada et al. ${ }^{[3]}$ reviewed the records of 154 children with NF1 and plexiform neurofibroma, and noted that patients with symptomatic plexiform neurofibroma had an increased occurrence of other NF1-related tumors. The most frequent indications for surgery included neurological, orthopedic, disfigurement and respiratory complaints. The extent of tumor resection correlated with outcome. Tumors in the head, neck and thorax had the highest recurrence. These studies indicate that surgical removal of plexiform neurofibroma is associated with high rates of acute and chronic morbidity. Recurrence is common when the resection could not be complete, the patient is young and in cases when the tumor invaded structures in the head, neck or thorax.

In the light of above studies ${ }^{[3,4]}$, our young patient with a symptomatic cervical plexiform neurofibroma has a high risk for recurrence. The complete removal of all microscopic tumor fragments from a location (e.g. the cervical region) with a high concentration of eloquent anatomical structures may not be always feasible, giving rise to the higher recurrence rates of tumors in such a location. In addition, the carrier status of the NF1 tumor suppressor gene mutation may predict a likely occurrence of other NF1-related benign or malignant tumors. Therefore, a continuous monitoring for recurrence and potential new tumor occurrence will be necessary for our patient with a histologically typical and relatively benign plexiform neurofibroma.

Unfortunately, the currently available pharmaceutical interventions, at best, would only delay recurrence, reduce the tumor size and slow disease progression ${ }^{[2]}$. Our patient is currently on interferon $\alpha$ therapy, and if it appears to be ineffective, we are prepared to switch her to imatinib. While a true disease modifying or curing drug is not yet available, some optimism may be drawn from recent reports on better characterizing molecular heterogeneity of NF1 tumors and from results of biologically targeted clinical trials ${ }^{[2,6]}$. An improved understanding of acquired molecular drivers playing key roles in tumor growth in addition to the inherited NF1 mutation(s) may identify correlates of long term outcomes as well as targets of more effective personalized treatment modalities much needed for the control of this complex disease.

\section{Conclusion}

This case represents clinical, imaging, histological and molecular characteristics of a large plexiform neurofibroma, and highlights the difficulties encountered during diagnostic work up and patient management. Our report underscores the utmost importance, while also illustrates the challenging nature of surgical resection of such a highly invasive tumor in an anatomically eloquent location. As long term morbidities are not uncommon, continuous monitoring for tumor recurrence and new tumor occurrence will be critical in our case. Currently, we only have a limited armamentarium of pharmaceutical agents. The long term outcome for our patient will be determined by the early detection of disease progression, early removal of new lesion (if feasible) and the availability of newer treatment modalities.

\section{Declaration of interest}

The authors report no conflicts of interest. The authors alone are responsible for the content and writing of the paper.

\section{Acknowledgements}

The authors' salaries have been supported by the Markusovszky University Hospital, Peterfy Sandor Hospital, and the University of Pecs. The genetic studies were performed at the Institute for Human Genetics, University of Pecs. The authors would like to acknowledge the outstanding imaging studies performed by the radiologists at the Markusovszky University Hospital. 


\section{References}

[1] Staser K, Yang F-C, Clapp DW. Pathogenesis of Plexiform Neurofibroma: Tumor-Stromal/Hematopoietic Interactions in Tumor Progression. Annual Review of Pathology: Mechanisms of Disease. 2012; 7: 469-495. Available from:

http://www.ncbi.nlm.nih.gov/pubmed/22077553. http://dx.doi.org/10.1146/annurev-pathol-011811-132441

[2] Gutmann DH, Blakeley JO, Korf BR, Packer RJ. Optimizing biologically targeted clinical trials for neurofibromatosis. Expert Opin Investig Drugs. 2013; 22: 443-462. Available from: http://www.ncbi.nlm.nih.gov/pubmed/23425047. http://dx.doi.org/10.1517/13543784.2013.772979

[3] Prada CE, Rangwala FA, Martin LJ, Lovell AM, Saal HM, Schorry EK, et al. Pediatric plexiform neurofibromas: impact on morbidity and mortality in neurofibromatosis type 1. J Pediatr. 2012 Mar; 160(3): 461-7. Epub 2011 Oct 11. http://dx.doi.org/10.1016/j.jpeds.2011.08.051

[4] Nguyen R, Ibrahim C, Friedrich RE, Westphal M, Schuhmann M, Mautner VF. Growth behavior of plexiform neurofibromas after surgery. Genet Med. 2013 Sep; 15(9): 691-7. Epub 2013 Apr 18. http://dx.doi.org/10.1038/gim.2013.30

[5] Kamra HT, Dantkale SS, Birla K, Sakinlawar PW, Bharia PH. Plexiform neurofibroma in the submandibular gland along with small diffuse neurofibroma in the floor of the mouth but without neurofibromatosis-1: a rare case report. Ecancermedicalscience. 2013 May 2; 7: 313. Print 2013. http://dx.doi.org/10.3332/ecancer.2013.313

[6] Thomas L, Mautner VF, Cooper DN, Upadhyaya M. Molecular heterogeneity in malignant peripheral nerve sheath tumors associated with neurofibromatosis type 1. Hum Genomics. 2012 Sep 4; 6: 18. http://dx.doi.org/10.1186/1479-7364-6-18 